\title{
Improving health care quality through
}

\section{culturally competent physicians: leadership and organizational diversity training}

This article was published in the following Dove Press journal:

Journal of Healthcare Leadership

I4 February 20II

Number of times this article has been viewed

\author{
Irwin B Horwitz' \\ Marilyn Sonilal ${ }^{2}$ \\ Sujin K Horwitz ${ }^{3}$ \\ 'Cameron School of Business, \\ University of St. Thomas, Houston, \\ TX, USA; ${ }^{2}$ School of Public Health, \\ University of Texas, Houston, TX, USA
}

Correspondence: Irwin B Horwitz Cameron School of Business, University of St. Thomas, 3800 Montrose Blvd., Houston, TX 77006, USA

Tel +l 7139425917

Email horwiti@stthom.edu

\begin{abstract}
The growing diversity of the population has resulted in substantial challenges for the US health care system. A substantial body of evidence has identified significant disparities in health care among culturally and ethnically diverse patients, irrespective of income, that negatively affects such factors as diagnostic precision, quality of care, adherence to healing protocols, and overall treatment outcomes. Diversity has also been shown to compromise the functionality of health care teams that are increasingly comprised of members with culturally different backgrounds, in which diversity produces misunderstanding and conflict. Many of the problems stem from a lack of cultural competence among both physicians and teams under their supervision. To reduce the numerous problems resulting from inadequate cultural competence among health care professionals, this article examines ways in which the issues of diversity can be effectively addressed in health care institutions. It is advocated that physicians adopt a proactive transformational leadership style to manage diversity because of its emphasis on understanding and aligning follower values which lie at the heart of diversity-related misunderstandings. It is also held that for leadership training among physicians to be fully effective, it should be integrated with organizational-wide diversity programs. By doing so, the complimentary effect could result in comprehensive change, resulting in substantial improvements in the quality of health care for all patients.
\end{abstract}

Keywords: leadership, diversity, health care, disparities, medical education

\section{Introduction}

Demographers have shown that the US population is growing increasingly diverse, and the ramifications on the health care system from this trend are significant. ${ }^{1}$ When patients of different ethnicities or races enter the system, they are often confronted with health care providers who either do not understand the cultural differences that influence their decisions or use stereotypes as a heuristic from which erroneous assumptions are made. Concurrently, as the patient population is growing more diverse, so is the health care workforce, which in turn engenders its own set of problems, ranging from miscommunication to intense relational conflict. Ironically, research has shown that if properly managed, not only many of the problems affecting both the diverse patient population and the health care workforce could be reduced, but actually many areas in the delivery of quality care could be improved..$^{2-4}$ One of the key drivers of such change lies with developing physician leadership skills coupled with cultural competence that have been traditionally overlooked in favor of technical medical training. As health care institutions and treatment protocols are often integrated and highly complex, the importance of having a unifying leader to work at coalescing diverse subordinates into 
high-performing teams is an absolute necessity. This work examines the problems and evaluates potential solutions to the vexing problems of diversity through the training of physicians in transformational leadership techniques in conjunction with institutional diversity training programs.

\section{Disparities in care for diverse patient populations}

Cultural competence in health care has been operationalized as the ability to provide high-quality care for patients with diverse values, beliefs, and behaviors, including the customization of service delivery and administration to meet the patients' social and cultural needs. ${ }^{5}$ Research in the health care field has shown that cultural competence affects the quality of health care for diverse patient populations as racial and ethnic minorities are often burdened with higher rates of disease, disability, and death and tend to receive a lower quality of health care than nonminorities, even when access-related factors, such as insurance status and income, are taken into account. ${ }^{6}$ Recently, two reports from the Institute of Medicine highlighted the persistent racial and ethnic disparities in the current US health care system and further stressed the importance of health care providers' cross-cultural competence to better address the needs of diverse patient populations and ultimately provide culturally competent, quality patient care. ${ }^{7,8}$

Unfortunately, the medical education of health care workers in general, and physicians in particular, fails to adequately prepare these professionals with the skills for competently administering cross-cultural care. For example, in one study comparing cross-cultural understanding and health care outcomes, the investigators observed that US medical education is grounded almost exclusively in monocultural western European values and traditions. ${ }^{9}$ Although it can be universally accepted that health care providers' interest is in overseeing the best care for patients, they are constrained by their own cultural background and may often uniformly address the situations of diverse patients within a framework myopically influenced by their own ethnocentric biases. The overall impact of overlooking important cultural differences can lead to substantial compromises in patient care. The various consequences of failing to incorporate cultural differences in medical practice have been found to increase the probability of miscommunication which will lead to diagnostic errors, important screening opportunities being missed, dangerous medication interactions, and large percentage of patients failing to adhere to clinical protocols and follow-ups. ${ }^{10}$

Flowing from other research, other negative effects flowing from a lack of cultural competence have been discovered.
In particular, understanding the cultural values of patients have been linked with the effectiveness of health counseling, which in turn affects the ability to develop effective health promotion campaigns; when cultural competence is lacking, both the prevention and management of diseases are significantly compromised. ${ }^{10}$ For example, one study found the quality of patient-physician communication experienced by African American patients was worse relative to White American patients, with physicians using a less patient-centered approach with African American patients. ${ }^{11}$ This is especially important as patient-centered communication has been demonstrated to be linked with greater patient adherence to treatment protocols, higher recall of medical information, increased participation in treatment decisions, and greater overall satisfaction with care. ${ }^{11}$ Similar results were reported in a study conducted of African American patients who reported experiencing less participatory physician visits than White Americans. In contrast, race-concordant relationships between physicians and patients were rated as more participatory. ${ }^{12,13}$

Further studies have highlighted the problematic effect of patient diversity on medical treatment regardless of income. For example, one longitudinal study examined the impact of racial and ethnic differences over 10 states on preventable hospitalization. ${ }^{14}$ Their results indicated that working age and elderly African American adults, as well as Hispanic children and elderly Hispanic patients, had a higher risk for a preventable hospitalization than White American patients even after adjustments were made for differences in health care needs, insurance coverage, socioeconomic status, and the availability of primary care. Other studies have shown similar disparities among ethnically diverse adolescents with psychiatric problems, ${ }^{15}$ Arab Americans having significant communication problems with health care workers, ${ }^{16}$ treating Latino and African American children with special needs, ${ }^{17}$ and providing access to mental health services among elderly patients of varying backgrounds. ${ }^{18}$ Additionally, problems have also been documented among biodemographically diverse homosexuals, ${ }^{19}$ with disparities particularly ubiquitous in the treatment of HIV/AIDS among minority groups, often leading these individuals to forgo screening and participation in clinical trials. ${ }^{20,21}$

The concurrence of research on the relationship between patient diversity and health disparities largely stemming from the lack of cultural competence in health care providers has called for diversity training of caregivers to improve the quality of medical care for varying ethnic and racial groups. However, one complicating factor for a health care workforce in attaining cross-cultural competence is that the demographic 
composition of health care workers is also changing with the shift in the overall population demographics. Although some researchers have argued that the increase in the diversity of health care workers could act to increase the cultural understanding of diverse patients, its success is far from given. $3,5,22,23$ Organizational research does not uniformly predict such a change would solve the problem and may even cause more problems than it solves. As there has been debate in this area, the ensuing section will compare the advantages and disadvantages of using the diversity within health care institutions to address cross-cultural patient care and examine why far more is needed to address health care diversity issues.

\section{Why increasing US health care workforce diversity is not enough?}

It has been argued that tailoring the diversity of an area's health care workforce to reflect their patient population to augment cultural competence, and in turn reduce disparities, represents a viable solution, and numerous scholars support this view. ${ }^{22-25}$ The rationale of matching workforce and patient diversity is intuitive: a diverse staff will have a greater understanding of cultural issues and will more effectively address the needs of a similar patient population. Some proponents of this reasoning have further held that by increasing diversity in the health care workforce, the additional benefits of improving access to high-quality care for the medically underserved, increasing the breadth and depth of the US health research agenda, and expanding the pool of medically trained executives and policymakers ready to take up leadership positions in the health care system of the future can be obtained. ${ }^{24} \mathrm{~A}$ further argument in favor of such targeted selection has been made that a staff, primarily composed of minorities, would be less likely to engage in discriminatory practices based on race and ethnicity than a majority-composed homogeneous staff. ${ }^{26}$

However, while the solution of attaining a specific mix of health care worker sounds good in theory, in practice, doing so would be highly problematic. For example, research has demonstrated that many patients have their own biases toward members of other ethnicities, as light-skinned patients, such as Latinos and Asians, erroneously considered caregivers with darker skin and less proficiency in English to be poorly educated. ${ }^{27}$ Even beyond having to overcome patients' own negative stereotypes, there may be substantially complex legal equal employment opportunity restrictions on using ethnicity as a hiring criteria, and further, even if a matching workforce were numerically achieved, given health care workers rotate in shifts, there would be no guarantee that the diversity of the provider a patient receives is the same as their own.
Qualitatively, different problems are likely to arise from the differences caused by the differing diversity of the health care team members themselves. A study on the communication effectiveness of diverse nursing care teams highlighted that effective communication is the integrative mechanism in high-performing teams, and concluded that 'in practice, communication processes in racially and ethnically diverse teams are likely to interfere with the team's ability to tap the inherent potential in their diversity and will increase conflict, reduce self-efficacy and lower team performance' ( $p$ 1406). ${ }^{28}$ The study also discovered that when team members cluster in their own identity groups, team performance is inhibited. Two themes of behavior emerged from the study: different perspectives and alternate realities. ${ }^{28}$ It was found that team members interpreted their own interactions with others on their team from vantage points grounded in their cultural perspectives, and such differences resulted in divergent views of the situational realities. The tendency to view things through an ethnocentric lens increased both conflict and miscommunication due to team member's inability to identify the root sources of their conflict and consequently impeded the development of pragmatic solutions. ${ }^{28}$

Without physicians who are not only experts in medical care, but also educated in value-based cultural leadership, treating and communicating increasingly diverse patients would be further compromised, and managing the differences of the increasingly diverse teams under their direction would create more miscommunication and problems than those exist currently. Numerous studies demonstrate the acquisition of leadership skills by physicians strongly facilitates communication and interaction with diverse patients and augments the effectiveness of their pedagogical and administrative roles over medical teams, thereby improving the overall treatment of diverse patient populations. ${ }^{3,7,23}$ In doing so, the best overall results are when such leadership training is accompanied by an organizational-wide diversity training initiative. It is, therefore, crucial that both physicians and health care workers become culturally competent and exhibit leadership to manage diversity for substantially improving the delivery of health care for all.

\section{The role of physicians as leaders in diversity management Physicians as leaders of diverse health care teams in cross-cultural patient care}

Because the delivery of health care in clinical environments is often highly complex, achieving optimal patient outcomes often necessitates the effective use and coordination of teams. ${ }^{29}$ 
Most frequently, such teams are rigidly hierarchical with primary and attending physicians charged with the responsibility of directing all caregivers in the coordination of a comprehensive treatment protocol. ${ }^{30}$ The purpose of this type of organizational structure is based on the premise that physicians are the most medically educated and knowledgeable about patient symptoms and conditions of all health care workers and are thus the most qualified to specify and modify specific medical regimens. Because of this, physicians are inherently placed in leadership positions, in which their authority flows from five primary power bases originally described by French and Raven. ${ }^{31}$

The first, expert power, flows from possessing a higher level of competence than other members of their organization or team. For example, when in the operating room, nurses obey orders from surgeons without hesitation due to their significantly higher medical proficiency. ${ }^{32}$ Legitimate power is that which is granted by the organization through establishing formal levels of authority, generally by position and/or rank within a position. Reward power stems from the ability to satisfy subordinate needs, and conversely, coercive power flows from the ability to punish followers by withholding or reducing things fulfilling their desires or needs. Often, these two types of power are held by those with legitimate power. The final base of authority, referent power, is achieved through an individual's charisma and willingness to employ that charisma to influence followers' deeply held beliefs. This is a particularly important source of power, as it is through this means that physician leadership can incorporate and appeal to the intrinsic values of followers and align the varying interests of individual caregivers into that of a team. ${ }^{33,34}$ However, as research on the effectiveness of charisma as a leadership style has been found to differ cross-culturally, its application needs to be tailored to suit the specific ethnic populations to when it is utilized. ${ }^{35}$

Unfortunately, despite the power physicians have from their medical expertise and formal institutional authority, physician-led medical teams are often characterized by high conflict and dysfunctional interaction. ${ }^{36}$ The rigid hierarchical organizational structure further compounds communication difficulties among team members, thereby leading to difficulties in command and coordination, and informal channels of information transfer develop..$^{30,37}$ Once communication breaks down, often conflict ensues and has been a well-documented literature, assessing problems in physiciannurse interactions. ${ }^{38-40}$ The resultant conflict between doctors and nurses can consequently lead to medication errors, unnecessary testing, and delayed diagnoses. ${ }^{41}$ Furthermore, once a pattern of such conflict becomes institutionalized over long periods of time, the mutual distrust and lack of respect between the parties negatively affect the decision-making quality of the health care providers by slowing the flow or introducing ambiguity into important patient information, as well as discounting the importance of such information when it comes from a party with whom the physician is in conflict. ${ }^{42}$ The hierarchal medical structure itself is, therefore, another problematic factor that causes miscommunication and conflict among diverse employees in work teams that undermine the ability to provide optimal health care to patients.

Furthermore, as today's health care workforce is highly diverse, without a high level of cultural understanding, communication by those from a different culture may be taken out of context or misunderstood by those from another culture. ${ }^{43,44}$ Indeed, this has been shown to be pronounced in modern workplaces when those with cultural differences communicate using e-mail, in which even simple grammatical violations may ferment distrust and create negative perceptions of the sender. ${ }^{45}$ These misperceptions, in turn, can become manifested in false stereotypes, thus further leading to problematic team relations. ${ }^{46}$ Although some studies have shown that for long-standing teams, such miscommunication is reduced over time ${ }^{44}$ and the effects of biodemographic differences may even have little to no effect on the quality of team member's work, ${ }^{47}$ minority members, who become stereotyped, may become inhibited from contributing potentially valuable input, and when they do, such opinions may be given little consideration. ${ }^{44}$ This can become detrimental in the administration of patient care, as often when diverse teams work functionally, they can spawn greater creativity than homogeneous teams. ${ }^{48,49}$

Until fairly recently, medical education has almost exclusively focused on the technical skills in physician training. As advances in medicine and patient treatment grew increasingly complex and interdependent, the delivery of care became increasingly team-based, while medical education remained largely grounded in the teaching traditions it had developed over the years, and leadership training largely ignored. ${ }^{50,51}$ However, a growing recognition of this problem has begun to resonate with medical educators, and calls for teaching leadership skills as a part of medical training has garnered growing support among physicians. ${ }^{52-63}$ As a result of this change in thinking, the Accreditation Council for Graduate Medical Education added the leadership skills of 'interpersonal skills and communication' and 'professionalism' to their list of core competencies. ${ }^{54}$ Although these two elements together do not constitute a full description of the qualities 
associated with broader definitions of leadership (which are numerous), these two in themselves are widely believed to be essential components of leadership, and particularly necessary to master, given the difficulty of leading diverse health care staff charged with treating a diverse patient population. Cohen, in his discourse on 'Finishing the bridge for diversity', called for his medical colleagues to take action for using leadership techniques to address the issues of diversity in medicine. ${ }^{55}$ In doing so, he strongly advocated that highquality health care and discovering medical solutions cannot be fully realized without strong leadership to augment efforts to increase cultural competence. The section below discusses the implications of developing physician leadership using transformational leadership for managing diverse work teams in delivering effective cross-cultural patient care.

\section{Leading diverse work teams through transformational leadership}

Leading teams comprised of highly diverse workers are fraught with managerial difficulties, especially as their primary mission is to practice medicine on an equally diverse patient population. One of the most difficult problems facing those who want to improve the performance of diverse health care teams in organizations is establishing the basis for trust in an environment where many established practices have led to an ingrained pattern of individual conflict over a long period of time. ${ }^{56}$ In order to do so, existing conflict must be acknowledged by all parties, and then, a greater understanding of the problems examined before a full resolution of the sources of conflict can be addressed. ${ }^{57}$ The added complexities associated with conflicts in teams high in cultural diversity are significantly more difficult to address than in homogeneous teams, as culturally and linguistically related misunderstandings may create or exacerbate a team conflict, and resorting to 'talking out' such problems ironically fail for the very reason they started. ${ }^{58}$

Team conflict has been an area widely researched by organizational scholars, and not all has been determined to be bad. Jehn developed a conflict model through, first, conducting a literature review and subsequently performed a qualitative analysis using observation and interviews. ${ }^{59}$ From this effort, she identified three primary forms of conflict that affects organizational groups: task-related, relationship-based, and process-based. Although an excessive amount of any of these conflict types were deemed to be detrimental to group performance, moderate levels of task-based conflict was found to produce such effects as generating and evaluating alternatives, encouraging constructive criticism, and the forthright exchange of opinions, all of which positively improved evaluation and decision-making. ${ }^{59}$ The finding was consistent with other studies, demonstrating that task-related conflict can enhance creativity through eliciting divergent viewpoints, and thus represents an advantage the diverse teams have relative to homogenous teams, provided the diverse team is properly managed. ${ }^{36,60}$ Other research has shown, though, that the relationship between task conflict and group productivity is curvilinear and highest when the conflict is moderate. ${ }^{61}$ In contrast, relationship-based conflict outcomes of bickering, low satisfaction, and decreased performance were reported. Because much of the conflict that occurs from biodemographic misunderstandings is relational or becomes relational, this provides additional rationale for proactively working toward having diversity education health care as part of ongoing professional training in health care settings. ${ }^{59}$ Thus, to effectively manage conflict arising out of diversity-related issues, leaders must minimize diversity-related relational conflict, while strategically allowing a moderate level of task-related conflict between diverse team members to enhance solution generation capability and decision-making quality.

Because of the deep-rooted problems and often intense emotional reactions individuals have in diversity-related misunderstandings, the type of leadership style employed becomes of utmost importance. Because of the underlying divisions, although not all the problems (such as language barriers) can be directly addressed, a value-oriented and integrative style of leadership can best facilitate optimal communication and understanding. Although a plethora of leadership theories and techniques have been developed and tested over many years, the most appropriate style to manage conflict and communication issues for diverse health care employee in cross-cultural patient care is transformational leadership. ${ }^{33,34}$ The taxonomy of transformational leadership is based on influencing followers through pursuing goals that fulfill their intrinsic needs. ${ }^{62}$ Although some of the power of a transformational leader is grounded in charisma (which as discussed is generally not highly effective for influencing culturally diverse individuals), its real focus is on profoundly influencing individuals through articulating a vision that promotes the followers' own, deeply held, intrinsic personal beliefs and values. ${ }^{63}$ Indeed, the values and ethics espoused by the leader who acts in a way that demonstrates willingness to put their followers' needs before their own makes it a style of leadership that goes far beyond charisma and actively aims to promote an understanding among the culturally diverse being led. ${ }^{63}$

In some cases, when tasks are routine and do not involve moral contemplation, transactional leadership is used to elicit 
follower behavior by extrinsic exchanges in value, like reward for performing a set of duties, instead. ${ }^{27,62}$ However, as has been demonstrated through this work, the issues that are problematic in managing diversity arise from long-held cultural norms and social values, and thus be best addressed, in large part, with transformational leadership techniques. ${ }^{29,64}$ There are four basic techniques which comprise transformational leadership model. ${ }^{65,66}$ When a set of employee values have been recognized to be of high importance, then the technique of inspirational motivation that employs motivational communication to demonstrate commitment to pursue such values is used. Idealized influence is a transformational technique utilized in two ways. First, as a means of developing strong bonds with workers on a personal and emotional level, and second, as role model, exhibiting behavior that is consistent with forwarding the values of the followers; seeing leaders perform behaviors they espouse serves to permeate the team or organization as a whole. Individual consideration is a third means of transformational leadership that specifically aims to help specific groups or individual followers satisfy their intrinsic needs, while intellectual stimulation is the fourth transformational style and works to generate creative thinking among followers by posing relevant problems and valuing their input.

Physicians placed in a leadership positions without a clear understanding of the intrinsic needs of their workers must default to leading through transactional methods, and by doing so, the problems associated with diversity mismanagement manifest in suboptimal health care delivery. For example, with employee teams and followers, physician leaders could opt to provide monetary rewards, or use threats of punishment for nonperformance, but in doing so, the root causes of conflict and misunderstanding flowing from cultural diversity would be unaddressed, thus allowing for relationship-based conflict and dysfunctional attitudes to fester and ultimately erode a functional organizational environment. In contrast, through the use of transformational leadership, physicians can be much more effective leaders of diverse workers. As it has been shown that doctors very often place the interests of their patients ahead of their own ${ }^{67}$ and most health care workers also place a high intrinsic value of aiding sick individuals, this underlying commonality can serve as a strong basis from which to anchor their leadership style with their follower's core values. ${ }^{68}$ Again, it cannot be stressed enough that for such leadership to be efficacious, it is of the utmost importance that the physician as leader displays behavior that exemplifies complete dedication for adhering to honest and ethical principles in dealing with their subordinates and patients and through their decision-making processes. ${ }^{69,70}$

Transformational leadership is predicated on dynamic interactions with followers which may change over time and varying situational outcomes. Physicians choosing to utilize this method need to conduct ongoing analyses of their own behavior and performance in medical situations, attempt to reduce reliance on algorithmic responses, and lead in nonprogrammatic ways that buttresses the experiential transformational process as a whole. ${ }^{71}$ These personal changes are held to be qualitatively more significant than simply a shift in preference for a leadership style and have been shown to be capable of producing deep changes in personal values, standards of evaluation, and increased motivation. ${ }^{72,73}$

Another leadership paradigm, identity-based leadership development, can be very complementary for physicians opting to lead using transformational leadership. Initially, based on work role identity research, ${ }^{74}$ this experientialbased view became more widely recognized in the late 1990s and early 2000 and has since garnered substantial academic support. ${ }^{75-77}$ The model holds that as leaders mature and develop, they change as a result of their experiences and become more open to challenging their old views and behaviors in favor of new ones. ${ }^{71}$ With ongoing experience, leaders grow to redefine their values and purpose in light of prioritizing things differently. In order to reap the full benefits from this method, physicians must be willing to engage in the self-introspection of their actions and experience outside the training forum. Thus, through experiences gained over time would engage in a continuous cycle of improvement in their leadership acumen. ${ }^{78}$ This effect may be especially pronounced when increase in an empathetic understanding of their followers (and patients) is experienced while undergoing diversity training. ${ }^{79}$

In the case of physicians, who have been shown to find ambiguity a significant barrier to the administration of highquality care, incorporating transformational leadership can be especially useful for helping to perform in environments high in complexity and ambiguity. ${ }^{80}$ The extent of complexity and tightly integrated systems in modern health care administration, and the resultant deleterious effects of medical errors, was extensive enough for Gaba to deem medical care as a 'high-hazard' industry. ${ }^{81}$ In tandem with developing transformational leadership, identity-based changes have been shown to also augment systems thinking and environmental analysis, which are critical for leaders facing problems rooted in systemic causes. ${ }^{82,83}$ Given that diversity affects the quality of care which both physicians and their teams can provide, 
learning to engage in systems thinking gives leaders added effectiveness in problem identification, decision-making, and alternative evaluation. ${ }^{84}$

\section{Instituting organizational diversity training programs Integrating leadership and team training}

Physician leadership training in itself is not enough to fully develop high-functioning cross-cultural medical teams. Rather, physicians must work with team members to coordinate their competencies and aim to include them in decisionmaking and afford them autonomy when appropriate. In order for physicians to gain the empathetic cross-cultural competence needed for developing high-performance teams using the transformational method, it is paramount that they gain a very thorough understanding of the cultures they serve. ${ }^{85}$ One means of doing so is to establish diversity training programs within the health care centers, with ongoing participation by physicians and other health care providers from their own teams and throughout the organization.

Although there are medical complexities that are necessary for the physician to solely direct because of their high expertise, for tasks where outcomes are dependent on coordinated team performance, allowing members to share in the decision-making process give them feelings of ownership, partnership, and accountability in the holistic care of patients. ${ }^{86}$ Diversity workshops and decision training are particularly valuable for medical teams, where shared knowledge, coupled with practice and feedback, is necessary to meet cross-functional demands for optimal outcomes. ${ }^{87,88}$ Physician leaders and other diverse team members, working together, can learn over time to overcome cultural differences that once acted as an impediment to cohesiveness, and through doing so, they can create a psychologically collectivistic mind-set among members, hence resulting in greater coordination and integration of care ${ }^{89,90}$ As research has indicated that goal priority is among the strongest motivators for group collectivism and physicians and followers hold the same intrinsic value on patients' welfare, this congruence of purpose strongly supports combining transformational leadership with team training.

Of particular importance to develop teams and individuals adept in dealing with diversity is to base training on improving overall 'cultural intelligence'. ${ }^{91}$ Formally proposed as a unique taxonomy of intelligence of individuals' ability to function in culturally diverse environments, ${ }^{92}$ it was held to be comprised of four distinct multidimensional factors: metacognitive intelligence representing the overall ability to comprehend and adjust mental models of cultural assumptions, cognitive intelligence which is the ability to recognize and gain familiarity with cultural traditions and norms usually through interaction, motivational intelligence being the drive and interest one has toward learning about acting in situations with cultural ambiguity, and behavioral intelligence is the ability to engage in cross-cultural communication through using appropriate verbal and nonverbal means that are culturally sensitive. ${ }^{91}$ In a series of three studies performed on these cultural dimensions by Ang et al, it was found that both metacognitive and behavioral intelligence were significantly related to task performance, while metacognitive and cognitive cultural intelligence were positively correlated with cultural judgment and decisionmaking. It was this latter finding that led the researchers to conclude that diversity training programs must extend beyond the usual cognitive training characteristic of such programs and additionally focus on building the metacognitive and behavioral intelligences as well as incorporating exercises, such as physical simulations, emotional engagement, and encouraging individual reflection in these programs. ${ }^{92}$

Indeed, such high-involvement exercises have shown success in building cross-cultural teams. The use of virtual patients in medical school and the use of patient-centered simulation in surgical training have both proven to be effective at fostering skills which promote greater diversity sensitivity in medical practice. ${ }^{93,94}$ Perhaps, a more intense simulated approach has been through the use of Crew Resource Training, which is a model taken from aviation training, and is geared for adapting to different situations, with unique and diverse team members under real-time conditions. ${ }^{95}$ In general, the reaction from such training among medical professionals has been mixed, with some finding it very useful and others difficult to generalize to medical settings. A mixed training method, incorporating a leadership program with cultural goal alignment support in a clinical traineeship program in a primary care setting, has also reported success. ${ }^{96}$ Although each of these programs may not have fully addressed diversity issues, it can be seen how each, in their own way, touch upon each of the four factors of cultural intelligence elucidated previously, and in doing so demonstrate the value of considering means by which to increase cultural intelligence in designing cultural competency programs.

\section{Integrating diversity training in the holistic delivery of cross-cultural care}

Gaining insight into the cultural patterns of different patient ethnicities is necessary for optimizing the treatment of diverse patients. For example, it has been shown that understanding the cultural traditions of the Latino community, such as 
the interaction of the cultural paradigms of familismo (value of extended family), machismo (predominantly male decision-making), and fatalismo (belief in fate), can influence treatment outcomes. ${ }^{97}$ Although doctors often must know where patients are in most pain for the purposes of diagnosis, the machismo expectations of Latino males may lead them to feign indifference to pain, whereas a white male may be more forthcoming. In such instances, physicians may need to look more closely for other nonverbal cues, indicating pain than when treating white males.

In contrast to Latino populations, individuals with East Asian ethnicities (eg, Korean, Chinese, and Japanese) are more concerned with preserving 'face', and thus act to limit embarrassment by avoiding to ask sensitive questions directly. ${ }^{52}$ As a result, physicians and health care staff treating members of these ethnicities may have greater success by posing questions and eliciting responses in a manner which allows patients to reveal personal information while preserving their dignity. Physicians and health care staff must also be aware that the cultural diversity can impact the way in which the patient describes the symptoms of specific illnesses. For example, because depression is widely stigmatized in Asian cultures, Asian patients with depression may report only the physical manifestations of their problem, such as weight loss and insomnia, and avoid discussing their emotional state altogether. ${ }^{98}$ Culturally sensitive health care professionals may then want to attempt to elicit such personal information circumspectly, like asking about stress levels at home and work or other such areas indicative of personal unhappiness. The development of professional cultural diversity training programs is an important means by which physicians and staff can work together to improve their knowledge and change their behavior to create more positive intercultural interactions with patients and each other.

Diversity training programs should begin by establishing dialog and interaction between physician leaders and employees' representative of the diverse composition of the workplace itself, with the aim to challenge stereotypes and educate each other as to their personal values, as well as concerns. ${ }^{99}$ Such programs benefit most by focusing on the similarities between groups as opposed to highlighting and confronting differences. ${ }^{43}$ Other work has also demonstrated the added value of programs transcend just addressing differences and work to create close bonds and mutual respect between those of different ethnic or racial backgrounds. ${ }^{100}$

Two types of programs that have enjoyed success in improving cultural competence are awareness training and skill-building training. In awareness training, individuals are introduced to the concepts of diversity and the reasons of why understanding cultural differences are clinically important, while skill-building training works to familiarize trainees on the nuances of cultural differences and how to utilize such knowledge in actual practice. ${ }^{101}$ These training types are not mutually exclusive and often most effective when combined.

One example of such synergy has been reported by an extensive study by the National Demonstration Project on transitioning family practices into patient-centered medical homes. ${ }^{102}$ In their study, a key underlying element was establishing a shared vision and, importantly, 'shared leadership'. In this model, both autonomy and accountability were shared among team members, a particular example of the emergence of a patient-centered home that enjoyed much success with this technique. A general conclusion of the study was there is a need for a shift in mental models with regard to individual roles in health care organizations; in such a conceptualization, not only is the doctor-patient relationship valued, but also relationships between staff and patients are valued. In such a model, the values discussed in transformational leadership are allowed to cascade down to other team members within the actual practice, thereby increasing personal investment and commitment to patients' care. ${ }^{102}$

It is, however, important to caveat that a potential drawback of cross-cultural training is erroneously assuming that an acceptable level of cultural competence has been attained simply by having physicians and staff attend required diversity education classes. Without sincere changes in worker and physician attitudes, the same behaviors that have become habit from practice over the years may not change or quickly reemerge. As discussed previously, from the onset, diversity training should not be considered to be an easy process. In some cases, diversity training sessions can lead to arguments, tense dialogs, and personal attacks. ${ }^{103}$ Emotions can run high, and heated disagreements may arise. Of critical importance is to allow the participants to vent and confront perceived inequities before encouraging reconciliation. In practice, the issues resolved in the training sessions should reduce relational conflict and foster greater interpersonal understanding. As a means of augmenting the process, some researchers have advocated the establishment of 'safe environments' where it is implicitly agreed that members can go, exchange ideas freely, argue, and debate openly, without fear of repercussion. ${ }^{104}$

Moreover, research has indicated that a lack of cultural competence is more related to health care workers' unwillingness to engage in critical self-introspection and 
reevaluation of their biases toward patients of different ethnicities, rather than fault with cross-cultural training interventions. ${ }^{105}$ Because such personal changes cannot be acquired through the didactic methods of reading textbooks and unstructured discussion alone, experts have argued that for such training to be effective, health care professionals must be taught through interacting with individuals' representative of the diverse patient populations they will treat. ${ }^{24}$ Diversity programs often involve high-impact hands-on training and thus need to be structured so both physicians and staff can apply learned concepts and skills on patients while concurrently assessing their cross-cultural effectiveness. ${ }^{28}$ Last but not least, the support of senior administrative management has been determined to be vital for such educational endeavors to be fully successful at creating institutions adept in diversity management. ${ }^{20,106}$

\section{Summary and conclusion}

The attainment of cultural competence is imperative for improving upon the serious problems of health disparities experienced by individuals due to their personal ethnography. A lack of cultural understanding has been shown to lead to making patient diagnosis more difficult and prone to error, patient inability to make informed choices over their own care, inhibit the establishment of a trusting doctor-patient relationship, and other such problems all of which both independently and interactively lead to suboptimal care. Compounding the problem is the diversity of the teams charged with treating the patients, who in addition may have their own difficulties communicating with diverse patients, also face the same problems in exchanges with diverse coworkers. The misunderstandings and conflicts, which often arise out of them, negatively act to impair the effectiveness of team operation and thus results in diminished quality treatment for all patients under their care. The two major recommendations proposed in this work to address diversity issues in both patients and health care teams are physician leadership and institutional diversity training.

The first, physician leadership, is the umbrella under which all other change initiatives should be deployed in providing effective cross-cultural patient care. Leadership efforts must be cohesive; beginning with support at the executive level, enacted in practice by physicians, and effectively diffused throughout all levels in the organization. Without organization-wide support and a commitment to allocate the necessary resources and create an infrastructure that promotes synergism between all integrated in the delivery of patient care, the ability to deal with the challenges of increasing diversity in health care settings would be substantially compromised. ${ }^{8}$ In order to address the inherent problems in treating diverse patients and managing diversity in the health care workforce, effective leadership must be at the helm of the effort. Nevertheless, until now, the mastery of leadership has not been an important domain of physicians, as medical care was not nearly as integrated or complex as in the modern era. To this point, leadership education has not been a formal part of medical school curriculums. However, as the growing body of evidence demonstrating that the treatment of diverse patients are being mismanaged, a change in medical education is beginning, including patient-focused simulation, the use of cross-cultural virtual patients, and inclusion of leadership and communication training necessary to reduce conflict and miscommunication in cross-cultural patient care. ${ }^{77-79}$ As complete medical reeducation is, however, not a realistic alternative for those already established in the health care sector, the motivation to change will necessarily have to come from those currently in practice who see and acknowledge such problems in their institution. Physician leaders could greatly facilitate this process by making a commitment to themselves, engage in diversity education, interact with greater understanding of patients and team members, and act as transformational leaders in their organizational settings.

The second, diversity training, should encompass all levels of health care staff to alleviate miscommunication and conflict inherent in cross-cultural patient care. In doing so, we also emphasized the role of physician leadership as a complementary means to augment the efficacy of diversity training in cross-cultural patient care. Although the training of health care professionals is essential to addressing the multitude of diversity-related problems, ultimately the origination of such change must begin with the full dedication of top administrators to support such organizational change and sincere commitment of physicians to actively participate in cross-cultural training, reflect on their current beliefs, and be willing to engage in the personal growth necessary to identify, articulate, and effectively improve their own ability to treat diverse patients and gain the skills to lead diverse teams through the use of transformational leadership.

Physicians must make the commitment to assume the role of change agents in the process to develop and lead culturally competent health care organizations. By doing so, in tandem with significant organizational diversity training efforts, substantial improvements in the health care system can be realized. The current ability to deal with diverse 
patient populations is fully unacceptable. The cross-cultural competence that is gained through the physician transformational process, in conjunction with improved team functioning, will translate into significantly enhanced care for many diverse patients currently marginalized. ${ }^{106,107}$ Physicians trained in cross-cultural care could establish closer rapports with such patients, elicit greater medical information, and interpret patient communication more accurately. It would be expected that an increase in compliance would follow as well, as patients would be increasingly receptive to the physician's care. ${ }^{108}$ Health care teams, comprised of diverse employees competent in providing cross-cultural care and under the leadership of physicians committed to developing team members' potential and promoting high ethical values within their teams, would together create dynamic and inclusive health care environments, where all patients would receive the best of care regardless of background.

\section{Disclosure}

The authors report no conflicts of interest in this work.

\section{References}

1. An older and more diverse nation by midcentury. US Census Bureau News. August 14, 2008. Available from: www.census.gov. Accessed January 14, 2011.

2. Chang S, Waid E, Martinec DV, Zheng B, Swanstrom LL. Verbal communication improves laparoscopic team performance. Surg Innov. 2008;15(2):143-147.

3. Gawande AA, Zinner MJ, Studdert DM, Brennan TA. Analysis of errors reported by surgeons at three teaching hospitals. Surgery. 2003; 133(6):614-621.

4. Yule S, Flin R, Paterson-Brown S, Maran N. Non-technical skills for surgeons: a review of the literature. Surgery. 2006;139:140-149.

5. Anderson LM, Scrimshaw SC, Fullilove MT, Fielding JE, Normand J. Task Force on Community Preventive Services. Culturally competent healthcare systems. A systematic review. Am J Prev Med. 2003; 24 Suppl 3:68-79.

6. Kennedy ME. The role of the federal government in eliminating health disparities. Health Aff (Millwood). 2005;24(2):452-458.

7. Nelson A. Unequal treatment: confronting racial and ethnic disparities in health care. J Natl Med Assoc. 2002;94(8):666-668.

8. Toward health equity and patient-centeredness: integrating health literacy, disparities reduction, and quality improvement. Institute of Medicine. 2009. Available from: http://www.iom.edu/Reports/2009/ Toward-Health-Equity-and-Paitent-Centeredness-Integrating-HealthLiteracy-Disparities-Reduction-and-Quality-Improvement-WorkshopSummary.aspx. Accessed January 14, 2011.

9. Kagawa-Singer M, Kassim-Lakha S. A strategy to reduce cross-cultural miscommunication and increase the likelihood of improving health outcomes. Acad Med. 2003;78(6):577-587.

10. Brach C, Fraser I. Reducing disparities through culturally competent health care: an analysis of the business case. Qual Manag Health Care. 2002; 10(4):15-28.

11. Engebretson J, Mahoney J, Carlson ED. Cultural competence in the era of evidence-based practice. J Prof Nurs. 2008;24(3):172-178.

12. Johnson RL, Roter D, Powe NR, Cooper LA. Patient race/ethnicity and quality of patient-physician communication during medical visits. Am J Public Health. 2004;94(12):2084-2090.
13. Cooper-Patrick L, Gallo JJ, Gonzales JJ, et al. Race, gender and partnership in the patient-physician relationship. JAMA. 1994; 282(6):583-589.

14. Gaskin DJ, Hoffman C. Racial and ethnic differences in preventable hospitalizations across 10 states. Med Care Res Rev. 2000;57 Suppl 1: 85-107.

15. Rue DS, Xie Y. Disparities in treating culturally diverse children and adolescents. Psychiar Clin North Am. 2009;32(1):153-163.

16. Aboul-Enein BH, Aboul-Enein FH. The cultural gap delivering health care services to Arab American populations in the United States. J Cult Divers. 2010;17(1):20-23.

17. Coker TR, Rodriguez MA, Flores G. Family-centered care for US children with special health care needs: who gets it and why? Pediatrics. 2010;125(6):1159-1167.

18. Solway E, Estes CL, Goldberg S, Berry J. Access barriers to mental health services for older adults from diverse populations: perspectives of leaders in mental health and aging. J Aging Soc Policy. 2010; 22(4): 360-378.

19. Cargill VA, Stone VE. HIV/AIDS: a minority health issue. Med Clin North Am. 2005;89(4):895-912.

20. Bell SA, Bern-Klug M, Kramer KW, Saunders JB. Most nursing home social service directors lack training in working with lesbian, gay, and bisexual residents. Soc Work Health Care. 2010;49(9): 814-831.

21. Sullivan PS, McNaghten AD, Begley E, Hutchinson A, Cargill VA. Enrollment of racial/ethnic minorities and women with HIV in clinical research studies of HIV medicines. J Natl Med Assoc. 2007;99(3): 242-250.

22. Borkowski N. Organizational Behavior in Health Care. Sudbury, MA: Jones and Bartlett Publishers; 2005.

23. Cooper LA, Powe NR. Disparities in patient experiences, health care processes, and outcomes: the role of patient-provider racial, ethnic, and language concordance. The Commonwealth Fund. 2004. Available from: http://www.commonwealthfund.org. Accessed March 25, 2010.

24. Cohen JJ, Gabriel BA, Terrell C. The case for diversity in the health care workforce. Health Aff (Millwood). 2002;21(5):90-102.

25. Calman N. Making health equality a reality: the Bronx takes action. Health Aff (Millwood). 2005;24(2):491-498.

26. Brach C, Fraser I. Can cultural competency reduce racial and ethnic health disparities? A review and conceptual model. Med Care Res Rev. 2000;57 Suppl 1:181-217.

27. Aries NR. Managing diversity: the differing perceptions of line managers, line workers, and patients. Health Care Management Rev. 2004;29(3):172-180.

28. Dreachslin JL, Hunt PL, Sprainer E. Workforce diversity: implications for the effectiveness of health care delivery teams. Soc Sci Med. 2000; 50(10):1403-1414

29. Donabedian A. Definition of Quality and Approaches to Its Assessment. Ann Arbor, MI: Health Administration Press; 1980.

30. Sexton JB, Thomas EJ, Helmreich RL. Error, stress, and teamwork in medicine and aviation: cross sectional surveys. $B M J$. 2000;320(7237):745-749. Available from: http://www.bmj.com/cgi/ content/full/320/7237/745. Accessed March 13, 2009.

31. French JRP, Raven BH. The bases of social power. In: Cartwright D, Zandler A, editors. Group Dynamics: Research and Theory. 2nd ed. New York, NY: Harper Row; 1960.

32. Denison DR, Sutton RI. Operating room nurses. In: Hackman J, editor. Groups That Work (and Those That Don't): Creating Conditions for Effective Teamwork. San Francisco, CA: Jossey-Bass; 1990.

33. Burns JM. Leadership. New York, NY: Harper and Row; 1978.

34. Bass BM. Leadership and Performance Beyond Expectations. New York, NY: Free Press; 1985

35. Ensari N, Murphy SE. Cross-cultural variations in leadership perceptions and attribution of charisma to the leader. Organ Behav Hum Decis Process. 2003;92(1-2):52-66. 
36. Horwitz SK, Horwitz IB, Barshes NR. Addressing dysfunctional relations among health care teams: improving team cooperation through applied organizational theories [book chapter]. In press.

37. Friedman DM, Berger DL. Improving team structure and communication: a key to hospital efficiency. Arch Surg. 2004;139(11):1194-1198.

38. Sutcliffe KM, Lewton E, Rosenthal MM. Communication failures: an insidious contributor to medical mishaps. Acad Med. 2004; 79(2):186-194.

39. Morrissey J. Dr. intimidation. Surly prescribers increase risk of errors. Mod Healthc. 2004;34(14):10.

40. Rosenstein AH, O'Daniel M. Disruptive behavior and clinical outcomes: perceptions of nurses and physicians. Am J Nurs. 2005;105(1):54-64.

41. Behal R, Finn J. Understanding and improving inpatient mortality in academic medical centers. Acad Med. 2009;84(12):1657-1662.

42. Larson JR Jr, Christensen C, Franz TM, Abbot AS. Diagnosing groups: the pooling, management, and impact of shared and unshared case information in team-based medical decision making. J Pers Soc Psychol. 1998;75(1):93-108.

43. Mannix E, Neale MA. What differences make a difference? The promise and reality of diverse teams in organizations. Psychol Sci Public Interest. 2005;6(2):31-55.

44. Levi D. Group Dynamics for Teams. 3rd ed. Los Angeles, CA: Sage; 2011.

45. Vignovic JA, Thompson LF. Computer-mediated cross-cultural collaboration: attributing communication errors to the person versus the situation. J Appl Psychol. 2010;95(2):265-276.

46. Marshall RS, Boush DM. Dynamic decision-making: a cross-cultural comparison of US and Peruvian export managers. J Int Bus Stud. 2001;32(4):873-893.

47. Horwitz SK, Horwitz IB. The effects of team diversity on team outcomes: a meta-analytic review of team demography. J Manag. 2007;33(6):987-1015.

48. Jackson SE. Team composition in organizational settings: issues in managing an increasingly diverse workforce. In: Worchel S, Wood W, Simpson JA, editors. Group Process and Productivity. Los Angeles, CA: Sage; 1992.

49. Edmondson AC, Nembhard IM. Product development and learning in project teams: the challenges are the benefits. J Prod Innov Manag 2009;26(2):123-138.

50. Guthrie MB. Challenges in developing physician leadership and management. Front Health Serv Manage. 1999;15(4):3-26.

51. Merry MD. Physician leadership. The time is now. Physician Exec. 1996;22(9):4-9.

52. Schwartz RW. Physician leadership: a new imperative for surgical educators. Am J Surg. 1998;176(1):38-40.

53. McAlearney AS, Fisher D, Heiser K, Robbins D, Kelleher K. Developing effective physician leaders: changing cultures and transforming organizations. Hosp Top. 2005;83(2):11-18.

54. Accreditation Council for Graduate Medical Education. http://www. acgme.org/acWebsite/RRC_280/280_coreComp.asp. Accessed September 21, 2010.

55. Cohen JJ. Finishing the bridge to diversity. Acad Med. 1997; 72(2):103-109.

56. Danjoux Meth N, Lawless B, Hawryluck L. Conflicts in the ICU: perspectives of administrators and clinicians. Intensive Care Med. 2009;35(12):2068-2077.

57. Lemieux-Charles L, McGuire WL. What do we know about health care team effectiveness? A review of the literature. Med Care Res Rev. 2006;63(3):263-300.

58. Glinow MAV, Shapiro DL, Brett JM. Can we talk, and should we? Managing emotional conflict in multicultural teams. Acad Manage Rev. 2004;29(4):578-592.

59. Jehn KA. A qualitative analysis of conflict types and dimensions in organizational groups. Adm Sci Q. 1997;42:530-557.

60. Klien KJ, Harrison DA. On the diversity of diversity: tidy logic, messier realities. Acad Manage Perspect. 2007;21(4):26-33.
61. Farh JL, Lee C, Farh CI. Task conflict and team creativity: a question of how much and when. J Appl Psychol. 2010;95(6):1173-1180.

62. Bass BM, Riggio RE. Transformational Leadership. 2nd ed. Mahwah, NJ: Lawrence Erlbaum; 2006.

63. Hughes RL, Ginnett RC, Curphy GJ. Leadership: Enhancing the Lessons of Experience. 2nd ed. Chicago, IL: Irwin; 1996.

64. Kearney E, Gebert D. Managing diversity and enhancing team outcomes: the promise of transformational leadership. J Appl Psychol. 2009;94(1):77-89.

65. Bass BM. From transactional to transformational leadership: learning to share the vision. Org Dyn. 1990;18(3):19-32.

66. Bass BM, Avilio BJ. Multifactor Leadership Questionnaire. 3rd ed. Palo Alto, CA: Mind Garden Inc; 2004.

67. Eastman JK, Eastman KL, Tolson MA. The relationship between ethical ideology and ethical behavior intentions: an exploratory look at physicians' responses to managed care dilemmas. J Bus Ethics. 2001;31(3):209-224.

68. Horwitz IB, Horwitz SK, Daram P, Brandt ML, Brunicardi FC, Awad SS. Transformational, transactional, and passive-avoidant leadership characteristics of a surgical resident cohort: analysis using the Multifactor Leadership Questionnaire and implications for improving surgical education curriculums. J Surg Res. 2008;148(1): 49-59.

69. Wilson DS, Redman RW, Potempa KM. A vision and compass for health care leadership: lessons from the migrant nurse resolution for recurrent nursing shortage. J Healthc Leadersh. 2010;2:91-96.

70. Rosenbloom AH, Jotkowitz A. The ethics of the hospitalist model. J Hosp Med. 2010;5(3):183-188.

71. Ibarra H, Snook SA, Ramo LG. Identity-based leader development. In: Nohria N, Khurana R, editors. Handbook of Leadership Theory and Practice. Boston, MA: Harvard University Press; 2010.

72. Joiner WB, Josephs SA. Leadership Agility: Five Levels of Mastery for Anticipating and Initiating Change. San Francisco, CA: Jossey-Bass; 2007.

73. Torbert WR. Action Inquiry: The Secret of Timely and Transforming Leadership. San Francisco, CA: Berrett-Koehler; 2004.

74. Ibarra H. Professional selves: experimenting with image and identity in professional adaptation. Adm Sci Q. 1999;44(4):764-792.

75. Brewer MB, Roccas S. Individual values, social identity and optimal distinctiveness. In: Sedikides C, Brewer MB, editors. Individual Self, Rational Self, Collective Self. Philadelphia, PA: Psychology Press; 2001.

76. Gelfand MJ, Erez M, Aycan Z. Cross-cultural organizational behavior. Annu Rev Psychol. 2007;58:479-514.

77. Cooper D, Thatcher SMB. Identification in organizations: the role of self-concept orientations in identification moves. Acad Manage Rev. 2010;35:516-538.

78. Schwandt DR. When managers become philosophers: integrating learning with sense making. Acad Manage Learn Educ. 2005;4(2): 176-192.

79. Bass BM. Does the transactional-transformational leadership paradigm transcend organizational and national boundaries? Am Psychologist. 1997;52(2):130-139.

80. Jung DI, Chow C, Wu A. The role of transformational leadership in enhancing organizational innovation: hypotheses and some preliminary findings. Leadersh Q. 2003;14(4-5):525-544.

81. Gaba DM. Structural and organizational issues in patient safety: a comparison of health care to other high-hazard industries. Calif Manage Rev. 2001;43(1):83-102.

82. Chrisman NJ. Extending cultural competence through systems change: academic, hospital and community partnerships. JTranscult Nurs. 2007; 18 Suppl 1:68S-76S.

83. Trofino J. Transformational leadership in health care. Nurs Manage. 1995;26(8):42-47.

84. Goeschel CA, Wachter RM, Pronovost PJ. Responsibility for quality improvement and patient safety: hospital board and medical staff leadership challenges. Chest. 2010;138(1):171-178. 
85. Majmudar A, Jain AK, Chaudry J, Schwartz RW. High-performance teams and the physician leader: an overview. J Surg Educ. 2010;67(4): 205-209.

86. Gorman JC, Cooke NJ, Amazeen PG. Training adaptive teams. Hum Factors. 2010;52(2):295-307.

87. Maizes V, Rakel D, Niemiec C. Integrative medicine and patientcentered care. Explore (NY). 2009;5(5)277-289.

88. Dierdorff EC, Bell ST, Belohlav JA. The power of 'we': effects of psychological collectivism on team performance over time. $J \mathrm{Appl}$ Psychol. October 4, 2010. [Epub ahead of print].

89. Price D, Howard M, Hilts L, et al. Interprofessional education in academic family medicine teaching units: a functional program and culture. Can Fam Physician. 2009;55(9):901, e1-e5.

90. James J, Green D, Rodriguez C, Fong R. Addressing disproportionality through undoing racism, leadership development, and community engagement. Child Welfare. 2008;87(2):279-296.

91. Early PC, Ang S. Cultural Intelligence: Individual Interactions Across Cultures. Palo Alto, CA: Stanford University Press; 2003.

92. Ang S, Van Dyne L, Koh C, et al. Cultural intelligence: its measurement and effects on cultural judgment and decision making, cultural adaptation, and task performance. Manage Organiz Rev. 2007;3:335-371.

93. Isenberg GA, Berg KW, Veloski JA, Berg DD, Veloski JJ, Yeo CJ. Evaluation of the use of patient-focused simulation for student assessment in a surgery clerkship. Am J Surg. September 17, 2010. [Epub ahead of print].

94. Fors UG, Muntean V, Botezatu M, Zary N. Cross-cultural use and development of virtual patients. Med Teach. 2009;31(8):732-738.

95. Helmreich RL, Schaefer HG. Team performance in the operating room. In: Bogner M, editor. Human Error in Medicine. Hillsdale, NJ: Lawrence Erlbaum; 1994.

96. Ruston A, Tavabie A. Fostering clinical engagement and medical leadership and aligning cultural values: an evaluation of a general practice specialty trainee integrated training placement in a primary care trust. Qual Prim Care. 2010;18(4):263-268.

97. Smith KA, Sudore LR, Perez-Stable JE. Palliative care for Latino patients and their families: whenever we prayed, she wept. JAMA. 2009;301(10):1047-1057, E1.
98. Kundhal KK, Kundhal PS. Cultural diversity: an evolving challenge to physician-patient communication. JAMA. 2003;289(1):94.

99. Battaglia B. Skills for managing multicultural teams. Cult Divers Work. 1992;4:4-12.

100. Trandis H. Culture and Social Behavior. New York, NY: McGraw-Hill; 1994.

101. Cox HT, Blake S. Managing cultural diversity: implications for organizational competitiveness. Acad Manage Exec. 1991;5(3):45-56

102. Nutting PA, Crabtree BF, Miller WL, Stewart EE, Stange KC, Jaén CR. Journey to the patient-centered medical home: a qualitative analysis of the experiences of practices in the National Demonstration Project. Ann Fam Med. 2010;8 Suppl 1:S45-S56.

103. Gardenshwartz L, Rowe A. The Managing Diversity Survival Guide: A Complete Collection of Checklists, Activities, and Tips/Book and Disk. Burr Ridge, IL: Irwin; 1994.

104. Edmondson A, Roloff K. Overcoming barriers to collaboration: psychological safety and learning in diverse teams. In: Salas E, Goodwin G, Burke C, editors. Team Effectiveness in Complex Organizations: Cross-Disciplinary Perspectives and Approaches. New York, NY: Routledge; 2008.

105. Tervalon M, Murray-Garcia J. Cultural humility versus cultural competence: a critical distinction in defining physician training outcomes in multicultural education. J Health Care Poor Underserved. 1998:9(2):117-125.

106. Jayne MEA, Dipboye RL. Leveraging diversity to improve business performance: research findings and recommendations for organizations. Human Resource Management. 2004;43(4):409-424.

107. Betancourt JR, Green AR, Carrillo JE, Ananeh-Firempong O 2nd. Defining cultural competence: a practical framework for addressing racial/ethnic disparities in health and health care. Public Health Rep. 2003;118(4):293-302.

108. Betancourt JR. Cultural competency: providing quality care to diverse populations. Consult Pharm. 2006;21(12):988-995.
Journal of Healthcare Leadership

\section{Publish your work in this journal}

The Journal of Healthcare Leadership is an international, peer-reviewed, open access journal focusing on leadership for the health profession. The journal is committed to the rapid publication of research focusing on but not limited to: Healthcare policy and law; Theoretical and practical aspects healthcare delivery; Interactions between healthcare and society and evidence-based

\section{Dovepress}

practices; Interdisciplinary decision-making; Philosophical and ethical issues Hazard management; Research and opinion for health leadership; Leadership assessment. The manuscript management system is completely online and includes a very quick and fair peer-review system. Visit http://www.dovepress. com/testimonials.php to read real quotes from published authors. 